101 ROLE OF CARDIAC AUTONOMIC FUNCTION IN PATHOPHYSIOLOGY OF PERMANENT AF

${ }^{1}$ Ahsan Khan, ${ }^{2}$ Rehan Junejo, ${ }^{3}$ Neil Thomas, ${ }^{4}$ James Fisher, ${ }^{5} \mathrm{G}$. Lip. ${ }^{1}$ University of Birmingham, Birmingham, UK; ${ }^{2}$ Manchester Metropolitan University; ${ }^{3}$ Institute of Applied Health Research, University of Birmingham; ${ }^{4}$ University of Auckland; ${ }^{5}$ Liverpool Centre for Cardiovascular Science, University of Liverpool and Liverpool Heart \& Chest

10.1136/heartjn-2021-BCS.100

Introduction Atrial fibrillation (AF) is the commonest abnormal heart rhythm with significant related morbidity and mortality. There is increasing evidence that abnormalities of the cardiac autonomic nervous system (ANS) are involved in the pathogenesis of AF. Exploring the ANS is possible through heart rate variability (HRV) evaluation. We aimed to investigate whether HRV is more abnormal in patients with permanent AF compared to paroxysmal AF.

Methods In a cross-sectional comparison, we studied two patient groups: permanent $\mathrm{AF}(\mathrm{n}=30)$ and paroxysmal $\mathrm{AF}$ $(\mathrm{n}=31)$. Time-domain, frequency-domain and non-linear measures of HRV were determined using eMotion Faros ECG sensor. Participant's breathing was controlled with a metronome. Data was analysed using SPSS software.

Results Time-domain and non-linear indices of HRV were significant higher in permanent $\mathrm{AF}$ group compared to paroxysmal AF (table 1). Permanent AF was the only independent

\begin{tabular}{|c|c|c|c|}
\hline & $\begin{array}{l}\text { Permanent AF group } \\
(\mathrm{n}=30)\end{array}$ & $\begin{array}{l}\text { Paroxysmal AF group } \\
(\mathrm{n}=31)\end{array}$ & $\mathrm{p}$ \\
\hline $\begin{array}{l}\text { Clinical } \\
\text { Demographics }\end{array}$ & Mean \pm SD / Median [IQR] & Mean \pm SD / Median [IQR] & \\
\hline Age, years & $70 \pm 8$ & $72 \pm 11$ & 0.64 \\
\hline $\begin{array}{l}\mathrm{CHA}_{2} \mathrm{DS}_{2}-\mathrm{VASC} \\
\text { score }\end{array}$ & $3[2-4]$ & $3[2-4]$ & 0.56 \\
\hline BMI $\left(\mathrm{kg} / \mathrm{m}^{2}\right)$ & $31.1 \pm 5.1$ & $31.0 \pm 6.3$ & 0.95 \\
\hline $\begin{array}{l}\text { Systolic BP } \\
\text { (mmHg) }\end{array}$ & $140[128-148]$ & 144 [134 - 153] & 0.24 \\
\hline $\begin{array}{l}\text { Diastolic BP } \\
(\mathrm{mmHg})\end{array}$ & $81 \pm 13$ & $76 \pm 15$ & 0.16 \\
\hline $\begin{array}{l}\text { Ejection fraction } \\
(\%)\end{array}$ & $55[55-62]$ & $62[55-68]$ & 0.22 \\
\hline $\begin{array}{l}\text { HRV } \\
\text { measurements }\end{array}$ & $\begin{array}{l}\text { Mean }[95 \% \mathrm{Cl}] \text { / Median } \\
{[95 \% \mathrm{Cl}]}\end{array}$ & $\begin{array}{l}\text { Mean }[95 \% \mathrm{Cl}] \text { / Median } \\
{[95 \% \mathrm{Cl}]}\end{array}$ & $\mathrm{p}$ \\
\hline $\begin{array}{l}\text { Mean heart rate } \\
\text { (bpm) }\end{array}$ & $75[68-82]^{a}$ & $66[60-71]^{a}$ & 0.03 \\
\hline SDNN (ms) & $90[82-113]^{b}$ & $39[28-59]^{b}$ & $<0.001$ \\
\hline rMSSD (ms) & $103[97-118]^{b}$ & $33[23-54]^{b}$ & 0.002 \\
\hline pNN50 (\%) & $64[61-70]^{\mathrm{b}}$ & $11[4-28]^{b}$ & $<0.001$ \\
\hline $\mathrm{SD} 1$ (ms) & $72[69-80]^{\mathrm{b}}$ & $23[17-38]^{b}$ & $<0.001$ \\
\hline SD2 (ms) & $104[96-137]^{b}$ & $51[35-65]^{b}$ & $<0.001$ \\
\hline
\end{tabular}

Normally distributed data are expressed as mean [95\% confidence intervals (CI)]. Identified by superscript a.Non-normally distributed data are displayed as median $[95 \% \mathrm{Cl}]$. Identified bysuperscript b. Normality test was performed using Shapiro-Wilk test. Statistical differences were tested using independent $\mathrm{t}$-test (for parametricdata) or Mann-Whitney $\mathrm{U}$ test (for non-parametric data). Significance $p>0.05$.

$\mathrm{AF}=$ atrial fibrillation; SDNN = standard deviation of all NN intervals; rMSSD = square root of the mean of the sum of thesquares of differences between adjacent NN intervals; pNN50 $=$ NN50 countdivided by the total number of all NN intervals predictor of HRV on multivariable analysis in this cohort of patients $(p=0.006)$.

Conclusions HRV indices were significantly higher in permanent AF compared to paroxysmal AF which may suggest pronounced cardiac autonomic influence in the pathophysiology of permanent AF.

Conflict of Interest None

\section{DETECTION OF ATRIAL HIGH-RATE EPISODES ON REMOTE MONITORING OF CARDIAC DEVICES: ARE WE FOLLOWING GUIDELINES TO DETERMINE THE NEED FOR ORAL ANTI-COAGULATION?}

Ronald Manorekang, Christopher Marshall, Vikrant Nayar. The Mid Yorkshire Hospitals NHS Trust, Wakefield, UK

\subsection{6/heartjnl-2021-BCS.101}

Background Atrial High-Rate Episodes (AHRE), as detected by implantable cardiac devices, increase stroke risk. Remote monitoring (RM) provides physicians with timely notification of such episodes through automatic downloads from home. The AHRE burden required to increase thromboembolic risk is unclear albeit the European Society of Cardiology (ESC) AF guidelines recommend initiating oral anticoagulation (OAC) in patients with at least a single episode of long duration $(\geq 1$ hour) and an overall 'high' daily burden of AHRE (total duration of all episodes), having considered the CHA2DS-VAS2C score. Moreover, device manufacturers are inconsistent with their nominal AHRE notification settings.

Aim In light of the ESC AF guidelines (2020), this study reviewed the current practise of RM for AHRE detection to determine OAC in patients with AHRE and CHA2DS-Vas2c $\geq$ 2 (Male) or 3 (Female).

Methods We retrospectively collected data from 50 patients with RM devices at a district hospital in the UK. Patients were selected to allow comparison between the 4 different manufacturers (Abbott, Biotronik, Boston Scientific, and Medtronic) used at the centre (figure 1). The latest AHRE notification alert settings and clinical data were obtained from electronic patient records. Table 1 shows the nominal settings.

Results Of the 50 patients, 50\% had dual chamber pacemaker (figure 2), mean age $74(\mathrm{SD} \pm 9$ ) years, 38 patients $(76 \%)$ had no documented history of atrial fibrillation at device implantation: 37 of them had an elevated CHA2DS-Vas2c score or previous TIA/stroke. Of these 37 patients, 33 patients had nominal RM settings with 8 of these patients later anticoagulated for AF; 4 patients had AHRE burden settings reduced to 1 hour, with one of these patients later anticoagulated for AF. DiscussionAbbott can alert for single, prolonged AHRE episodes in accordance with ESC guidance. However, episode detection is nominally set at 3 hours. AHRE burden can be determined by all manufacturers and may be used solely (without supporting guidance) in the absence of single episode alerts, pending robust trial data to conclusively alter the current OAC recomendation. Also, it is apparent that RM nominal settings are not actively altered by device implanting physicians but this may change as a result of the recently revised ESC AF guidance.In our cohort, 37 patients without a history of $\mathrm{AF}$ at device implantation were at significant risk of thromboembolism in the event of 


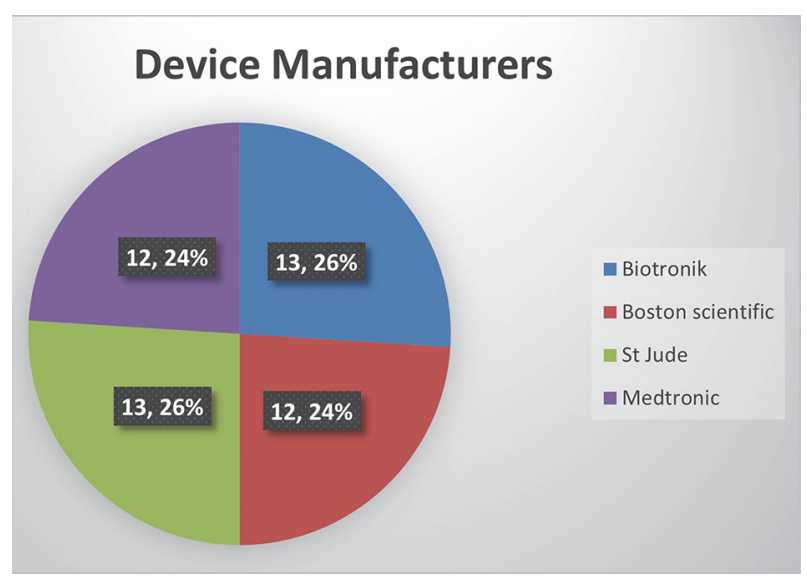

Abstract 102 Figure 1

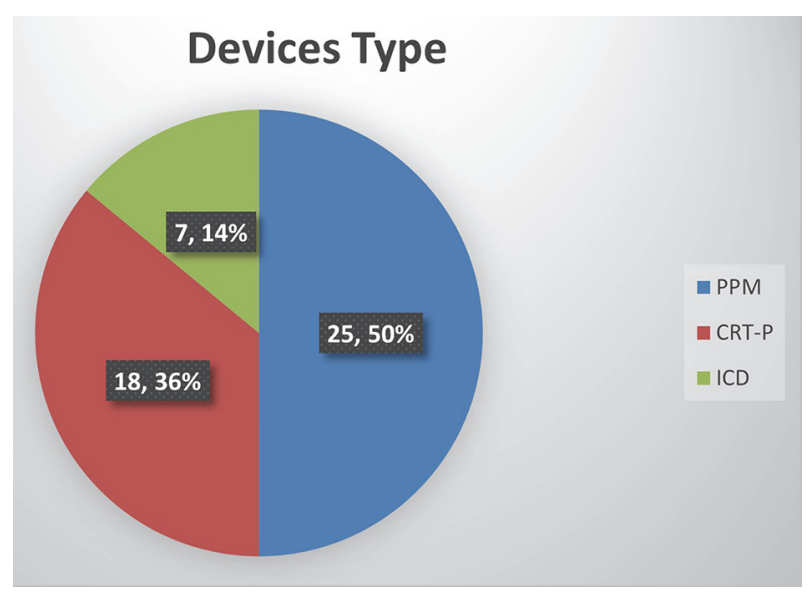

Abstract 102 Figure 2

Abstract 102 Table 1 RM nominal settings for AHRE alerts between different device manufacturers

\begin{tabular}{lllll}
\hline & $\begin{array}{l}\text { Single Episode } \\
\text { (On/Off) }\end{array}$ & $\begin{array}{l}\text { Single Episode } \\
\text { Duration }\end{array}$ & $\begin{array}{l}\text { Daily Burden } \\
\text { (On/Off) }\end{array}$ & $\begin{array}{l}\text { Daily Burden } \\
\text { Duration }\end{array}$ \\
\hline Abbott & On & $3 \mathrm{hr}$ & On & $6 \mathrm{hr}$ \\
Biotronik & N/A & N/A & On & $6 \mathrm{hr}$ \\
Boston & N/A & N/A & On & $0 \mathrm{hr}$ \\
Scientific & & & & \\
Medtronic & N/A & N/A & Off & $6 \mathrm{hrs}$ \\
\hline
\end{tabular}

developing AF. It is possible that the 9 patients on OAC may have initiated OAC sooner if significant AHRE been detected earlier. Similarly, limitations of AHRE detection and a failure by physicians to alter RM nominal settings potentially limited identification of other patients suitable for OAC.

Conclusions Guideline-directed AHRE detection settings are under-utilised even though most patients with implantable cardiac devices are at potentially high risk of developing thromboembolic events.

Conflict of Interest None

\section{3 \\ PAINFUL LEFT BUNDLE BRACH BLOCK SYNDROME AT REST TREATED WITH HIS-BUNDLE PACING}

${ }^{1}$ Alex Touze, ${ }^{2}$ Javed Iqbal, ${ }^{2}$ Tim Edwards, ${ }^{2}$ Holly Cousins. ${ }^{1}$ Portsmouth Hospitals University NHS Trust, Portsmouth, UK; ${ }^{2}$ Dorset County Hospital NHS Foundation Trust

\subsection{6/heartjnl-2021-BCS.102}

Introduction Painful left bundle branch block (LBBB) syndrome is a rare condition characterised by chest pain associated with intermittent LBBB, in the absence of significant coronary artery disease. Its prevalence, mechanism and management are not well described in the literature [1]. An ischaemic substrate has been postulated but largely disregarded in favour of a 'ventricular dyssynchrony' theory [2]. Nevertheless, most described cases occur on exertion [1], with only a single case of the condition occurring at rest being described [3]. Pacing has been used to successfully treat the condition via a range of modalities, including right ventricular pacing [4], cardiac resynchronisation therapy [4][5] and His-bundle pacing (HBP) [6][7]. We present a further patient who experienced the syndrome at rest, and successfully treated with HBP.

Case Presentation A 64 year old lady presented to the emergency department reporting acute onset cardiac sounding chest pain. An ECG revealed evidence of LBBB. Blood tests were performed but the results were not available before further investigations. The patient was immediately taken to the cardiac catheter lab for primary percutaneous coronary intervention. An angiogram revealed no significant coronary artery disease and no percutaneous intervention was performed. A CT-aorta was unremarkable. Her troponin was normal on serial measurements and the remainder of her blood tests were unremarkable. A repeat ECG when she was pain free revealed sinus rhythm with narrow QRS complexes and first degree heart block. An echocardiogram showed normal cardiac structure and function. The patient was moved to the coronary care unit and placed on cardiac monitoring. Over the next 24 hours the nursing staff observed and documented around 20 episodes of LBBB that coincided abruptly with the patient's reports of chest pain. During periods when her heart rate was less than 60 beats per minute (bpm) she had a narrow QRS interval, but when her heart rate increased to over $60 \mathrm{bpm}$, she developed LBBB. Her chest pain was debilitating and difficult to manage. Her baseline hypotension (90/50) and borderline bradycardia $(45-55 \mathrm{bpm})$ limited the use of chronotropic medications.

The diagnosis of painful LBBB syndrome was made and after considering the available literature, the decision was made to offer the patient a DDDR pacemaker with Hisbundle pacing, an intervention that has been recognised to offer effective symptomatic relief [6][7]. A DDDR pacemaker with HBP was implanted, achieving non-selective His-bundle pacing. Electrophysiology studies performed at the time revealed a narrow QRS complex at rates less than $60 \mathrm{bpm}$ with no chest pain. This was also achieved during non-selective His-bundle pacing at rates greater than $60 \mathrm{bpm}$. However, when AAI pacing was performed at rates above $60 \mathrm{bpm}, \mathrm{LBBB}$ was induced and the patient complained of chest pain.

The procedure was successful and afforded the patient immediate and persistent relief. She was discharged the next morning and at her one month follow up these positive results persisted.DiscussionThis case of painful LBBB syndrome is only the second described occurring at rest and offers 